Copyright (C) 2014 by Academic Publishing House Researcher

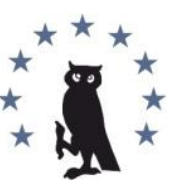

Published in the Russian Federation

European Researcher

Has been issued since 2010.

ISSN 2219-8229

E-ISSN 2224-0136

Vol. 86, No. 11-1, pp. 1937-1943, 2014

DOI: 10.13187/er.2014.86.1937

www.erjournal.ru

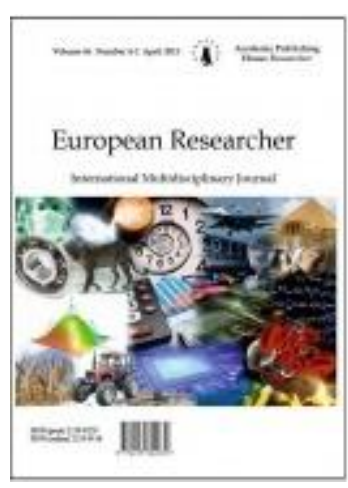

Экономические науки

Economic sciences

UDC 332.01

\title{
Information Asymmetry as a Risk Factor
}

Viktor Ya. Tsvetkov

Moscow State Technical University of Radio Engineering, Electronics and Automation (MSTU MIREA), Russian Federation

Doctor of Technical Sciences, Professor

E-mail: cvj2@mail.ru

\section{Abstract}

This paper explores information asymmetry as the cause of risks in decision making. The author describes the types of information asymmetry as a risk factor; describes the types of risk arising under different information asymmetries; describes the methods for minimizing such risks; brings to light the principal-agent issue; analyzes the principles of minimizing risks in the event of this issue arising; illustrates the application of special information models for minimizing risks in this issue; describes the cascade method for minimizing risks in decision making under information asymmetry.

Keywords: management; decision making; risks; information; information asymmetry; principal; information interaction; risk minimization.

\section{Введение}

В теории рисков различают три основных понятия $[1,2,3]$ : фактор риска, вид рисков, потери от наступления рисковых событий. Фактор риска является определяющим, поскольку он задает вид риска и служит основой оценки потерь. В условиях глобализации и информатизации общества [4] все большую роль играют риски связанные с информацией и информационными технологиями. Одним из факторов риска, негативно влияющих на рынок и принятие решений, является информационная асимметрия [5, 6, 7].

Информация является основой для принятия решений. Не информированность и информационная неопределенность влекут неверное принятие решений и в итоге обуславливают потери. Эти факторы могут быть обусловлены внутренними причинами отсутствие ресурсов для поиска качественной информации. Эти факторы могут быть обусловлены внешними причинами: рост объемов управленческой информации, рост сложности управленческой информации, проблема больших данных [8]. Информационная асимметрия как фактор риска может быть обусловлена всеми перечисленными причинами. Она может быть следствием не информированности и следствием неопределенности. Все это делает актуальным анализ информационной асимметрии как фактора риска при принятии 
решений и выработку рекомендаций для снижения этого риска. В аспекте принятия решений информационная асимметрия может быть рассмотрена как информационная ситуация связанная с информационной неопределенностью или не информированностью.

Виды информационной асимметрии как фактора риска. Информационная асимметрия как следствие не информированности достаточно подробно рассмотрена в литературе [5, 6]. Ее связывают в первую очередь с работами Дж. Акерлофа [7]. Применительно к проблеме рисков эти исследования можно условно разделить на две группы: анализ рисков принятия решений из-за некачественной информации и изучение возможности минимизации этих рисков. При анализе этих групп следует различать информирование и информационное взаимодействие [9].

В современных условиях профессионал в области сбора и обработки информации, который еще и является специалистом в предметной области, быстрее соберет качественную информацию, чем непрофессионал. Ограниченность в таких специалистах в большинстве фирм приводит к тому, что на практике широко используют посредников по получению специальной информации, необходимой для принятия решений и анализа ситуации на рынке. Существуют специальные организации, поставляющие экономическую аналитическую информацию для широкого круга организаций. Это технологии информирования.

Для минимизации риска не информированности потребитель вынужден нести транзакционные издержки на информационное обслуживание, которые, однако, ниже потерь вызванных неправильным принятием решения.

Как отмечено во многих работах [7] информационная асимметрия не возникает при статистическом характере продукции. Продавец и покупатель в этой ситуации одинаково информированы о свойствах продукции. При индивидуальном товаре продавец информирован больше чем покупатель и возникает информационная асимметрия, обусловленная не информированностью покупателя в сравнении с продавцом. Возникает риск принятия ошибочного решения относительно качества приобретаемого продукта.

Для минимизации этого риска потребитель вынужден нести транзакционные издержки [10] на информационное взаимодействие с посредником агентом. Это снижает риск ошибочных решений, однако приводит к новому виду асимметрии, или проблеме, которую называют проблемой «агент - принципал».

При приобретении нового оборудования, которое требует специальной подготовки и специальных знаний возникает информационная асимметрия, обусловленная когнитивным разрывом [11] между возможностью потребителя и требованиями эксплуатации оборудования. Возникает риск ошибочного решения из-за не компетенции потребителя. Когнитивный разрыв является характерным примером информационной асимметрии в информационных технологиях.

Для минимизации этого риска потребитель вынужден нести транзакционные издержки на информационное взаимодействие с посредником, который может работать на таком оборудовании. В альтернативном варианте потребитель должен нести издержки на обучение и подготовку по эксплуатации такого оборудования [12].

В условиях нестабильности ситуации внешней среды возникает информационная неопределенность, которая приводит к информационной асимметрии, обусловленной информационной неопределенностью. Возникает риск принятия ошибочного решения из отсутствия качественной информации. Для минимизации этого риска потребитель вынужден нести транзакционные издержки на информационное взаимодействие с аналитиком. В работе [13] такой вид информационной асимметрии называют асимметрией по «ситуации». Для минимизации этого риска потребитель также вынужден нести издержки на резервирование в случае возможных потерь. В случае информационной неопределенности ЛПР вынужден принимать решение по этапам с контролем решения на каждом этапе. Это по существу означает поэтапное информационное взаимодействие [14].

Информационные методы решения проблемы «агент принципал». Как показывает анализ, во многих случаях информационная асимметрия связана с деятельностью посредников, что создает проблему «агент принципал». В этом случае приходится иметь дело с информационным взаимодействием [9], а не получением информации. 
Исследование проблемы «агент принципал» осуществляется не менее 40 лет $[15,16]$. Разработан ряд подходов к решению этой проблемы. Холмстром и Милгром [17] предложили четыре принципа минимизирующие риски, возникающие при этой проблеме. Эти принципы включают: принцип информативности, принцип интенсивной стимуляции, принцип мониторинга интенсивности деятельности агента, принцип эквивалентной компенсации.

Принцип информативности в переводе на язык информатики и информационного управления можно интерпретировать как «принцип качественной информированности». По Холмстрому [17] мерой производительности агента является получение и предоставление принципалу информации необходимого качества для принятия решений. Это условие должно быть включено в договора компенсации. Данное условие включает в себя, например, относительную оценку эффективности деятельности агента по отношению к другим, подобным агентам. Относительная или интегральная оценка деятельности агента уменьшает случайные факторы его действий. При удалении экзогенных источников случайности работы агента, возникает возможность большей ответственности агента за результат работы и, главное, возрастет его ответственность за возникновение риска из-за некачественной информации. Это стимулирует агента к получению для принципала качественной информации.

Принцип интенсивной стимуляции утверждает, что оптимальная интенсивность деятельности агента зависит от четырех факторов: инкрементная прибыль за счет дополнительных действий, точность, с которой мероприятия оцениваются, толерантность агента к риску и реакция агента на стимулирование его деятельности.

Принцип мониторинга интенсивности деятельности агента является дополнением ко второму принципу. Он включает мониторинг ситуаций, в которых оптимальным является интенсивность стимулов действий агента. На основе такого мониторинга производится оценка стимулирующих факторов действий агента. Работодатели могут выбирать из «меню» факторы, обеспечивающие эффективность стимулирования действий агента. Такой мониторинг является средством сокращения неэффективных действий агента и ведет к уменьшению рисковых ситуаций.

Последний принцип эквивалентной компенсации достаточно прост и объективен. Но он сложен в реализации, поскольку в свою очередь создает информационную асимметрию, которую называют «проблемой царя Соломона» $[7,13]$. Эта проблема состоит в том, что исполнитель лучше знает свою работу, чем работодатель. Поэтому работодатель часто не в состоянии оценить адекватно работу исполнителя и особенно сравнить работу двух исполнителей.

Этот принцип утверждает, что деятельность агента должна быть эквивалентно оценена с точки зрения компенсации его затрат. При этом необходимо принимать во внимание как финансовые затраты и их компенсацию, так и нефинансовые компенсации, такие как моральные поощрения. Другими словами должны включаться социальные факторы оценки и поощрения деятельности агента. Возможны и неверные варианты такого принципа. Например, если преподаватель вознаграждается за результаты тестов студентов, то методика обучения может быть направлена на успешную сдачу тестов, а не на получение профессиональных знаний. Такая политика в результате снижает качество образования и увеличивает риск выпуска некомпетентных специалистов.

В целом совокупность этих принципов приводит к использованию информационного анализа и информационных моделей, таких как модель информационной ситуации [18], управление с выбором цели [19] и другие.

Каскадный метод минимизации риска принятия решений при информационной асимметрии. Каскадный метод широко применяется при решении разнообразных задач управления и проектирования. Он применяется при создании проектов и при оценке жизненного цикла проекта или продукта [20]. Как правило, метод применяется при наличии сложности [21] или неявного знания [22] в проекте и в модели жизненного цикла. Суть метода в переходе к поэтапному (инкрементному) решению. На рис. 1 показана структура каскадного метода принятия решений, минимизирующая риски информационной асимметрии. 
Минимизация рисков осуществляется за счет разбиения общего решения на этапы и введения контроля на каждом этапе за результатом промежуточного решения. На рис.1 условно приведены три этапа. Однако их количество определяется сложностью [21] принимаемого решения и может быть более трех.

Сплошными линиями показаны информационные потоки, отражающие прямые решения. Пунктирными линиями показаны информационные потоки обратных связей, вносящие коррекцию в принимаемые решения. Пунктирные линии создают потоки транзакционных издержек. Этот механизм работает циклично на каждом этапе принятия решений. Отсюда чем больше этапов, тем больше циклов анализа решения и тем больше транзакционные издержки.

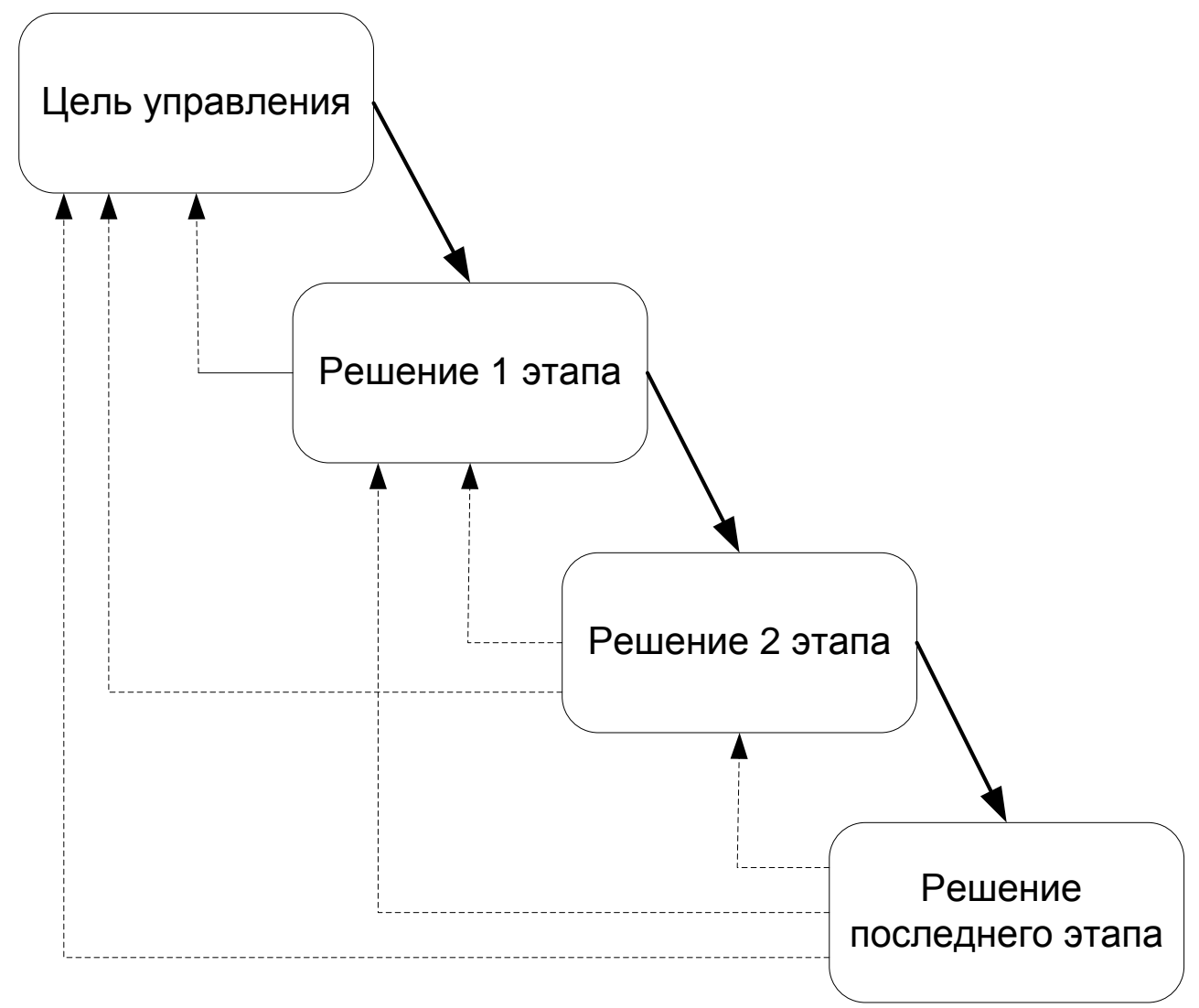

Puc. 1. Каскадный метод принятия решений

Информационная асимметрия создает неопределенность в принятии решений. Поэтому информационные потоки обратных связей выполняют функции информирования и информационного воздействия на первоначальные этапы принятия решения. Это уменьшает информационную асимметрию, существующую при первоначальном принятии решения.

Если информация, получаемая по информационным потокам обратных связей, не требует корректировки принимаемых решений, процесс принятия решений переходит к следующему этапу. Если информация, получаемая по информационным потокам обратных связей, требует корректировки принимаемых решений, процесс принятия решений возвращается к исходному этапу и в первоначальное решение вносится коррекция. Такой циклический процесс повторяется до снижения риска до приемлемого уровня и принятия решения с минимальным риском.

\section{Заключение}

Информационная асимметрия как фактор риска постоянно появляется в новых формах, что требует совершенствования механизмов ее обнаружения уменьшения и 
уменьшения рисков, обусловленных асимметрией. Информационная асимметрия создает когнитивный разрыв [11] и дихотомию [23] между субъектами информационных взаимодействий. Этим снижается эффективность взаимодействий и тормозятся информационные процессы. Основные принципы уменьшения информационной асимметрии: информирование и информационное взаимодействие, принимающие разные формы. Современная проблема минимизации рисков информационной асимметрии связана с большим количеством экспертного оценивания связанного как с четырьмя принципами, описанными Холмстром и Милгром [17], так и с традиционным отставанием руководителей в оценке деятельности своих сотрудников. Как ответная реакция это порождает антагонизм сотрудников и создает информационную асимметрию как результат неправильно принятия решений. Каскадный метод позволяет ее устранять, но при большом числе этапов существенно возрастают транзакционные издержки. Поэтому данное направление остается открытым для дальнейших исследований.

\section{Примечания:}

1. Boehm B.W. Software risk management: principles and practices // Software, IEEE. 1991. T. 8. №. 1. p. 32-41.

2. Chapman C., Ward S. Project risk management: processes, techniques and insights. John Wiley, 1996.

3. Reason J.T., Reason J.T. Managing the risks of organizational accidents. Aldershot : Ashgate, 1997. T. 6.

4. Цветков В.Я. Глобализация и информатизация // Информационные технологии. 2005. №2. c. 2-4.

5. Dunk A.S. The effect of budget emphasis and information asymmetry on the relation between budgetary participation and slack // Accounting review. 1993. p. 400-410.

6. Aboody D., Lev B. Information asymmetry, R\&D, and insider gains // The journal of Finance. 2000. T. 55. №. 6. p. 2747-2766.

7. Akerlof, George A. (1970). "The Market for 'Lemons': Quality Uncertainty and the Market Mechanism". Quarterly Journal of Economics (The MIT Press) 84 (3): 488-500.

8. V.Ya. Tsvetkov, A.A. Lobanov. Big Data as Information Barrier // European Researcher, 2014, Vol.(78), № 7-1. p. 1237-1242.

9. V.Ya. Tsvetkov. Information interaction // European Researcher, 2013, Vol.(62), № 11-1, p. 2573-2577.

10. Dyer J. Effective interfirm collaboration: how firms minimize transaction costs and maximize transaction value. 2002.

11. V.Y. Tsvetkov. Information Interaction as a Mechanism of Semantic Gap Elimination // European Researcher, 2013, Vol.(45), № 4-1, p. 782-786.

12. Цветков В.Я. Транзакционные издержки при внедрении информационных технологий и систем // Современные проблемы науки и образования .2008. №4. с. 114- 116.

13. Оболяева Н.М. Устранение информационной асимметрии как инструмент повышения качества образования // Известия высших учебных заведений. Геодезия и аэрофотосъемка. 2012. №6. с. 123-124.

14. Цветков В.Я. Информационное управление. LAP LAMBERT Academic Publishing GmbH \& Co. KG, Saarbrücken, Germany 2012, $201 \mathrm{c}$.

15. Grossman S.J., Hart O.D. An analysis of the principal-agent problem // Econometrica: Journal of the Econometric Society. 1983. p. 7-45.

16. Cvitanić J., Zhang J. Principal-Agent Problem // Contract Theory in Continuous-Time Models. Springer Berlin Heidelberg, 2013. p. 3-6.

17. Holmstrom B., Milgrom P. Multitask principal-agent analyses: Incentive contracts, asset ownership, and job design // Journal of Law, Economics, \& Organization. 1991. p. 24-52.

18. V.Ya. Tsvetkov. Information Situation and Information Position as a Management Tool // European Researcher, 2012, Vol.(36), № 12-1, p. 2166-2170.

19. V.Ya. Tsvetkov. Multipurpose Management // European Journal of Economic Studies 2012, Vol.(2), № 2 p. 140-143. 
20. Wen Y.M., Lu B.L. A cascade method for reducing training time and the number of support vectors // Advances in Neural Networks-ISNN 2004. Springer Berlin Heidelberg, 2004. p. $480-486$.

21. V.Ya. Tsvetkov. Complexity Index // European Journal of Technology and Design, 2013, Vol.(1), № 1, p. 64-69.

22. Цветков В.Я. Анализ неявного знания // Перспективы науки и образования, 2014. №1. C. 56-60.

23. Tsvetkov V.Ya. Dichotomous Systemic Analysis. Life Science Journal 2014; 11(6). P. 586-590.

\section{References:}

1. Boehm B.W. Software risk management: principles and practices // Software, IEEE. 1991. T. 8. №. 1. p. 32-41.

2. Chapman C., Ward S. Project risk management: processes, techniques and insights. John Wiley, 1996.

3. Reason J.T., Reason J.T. Managing the risks of organizational accidents. Aldershot : Ashgate, 1997. T. 6.

4. Tsvetkov V.Ya. Globalization and information // Information Technology. 2005. №2. p. 2-4.

5. Dunk A. S. The effect of budget emphasis and information asymmetry on the relation between budgetary participation and slack // Accounting review. 1993 . p. 400-410.

6. Aboody D., Lev B. Information asymmetry, R\&D, and insider gains // The journal of Finance. 2000. T. 55. №. 6. p. 2747-2766.

7. Akerlof, George A. (1970). "The Market for 'Lemons': Quality Uncertainty and the Market Mechanism". Quarterly Journal of Economics (The MIT Press) 84 (3). p. 488-500.

8. V.Ya. Tsvetkov, A.A. Lobanov. Big Data as Information Barrier // European Researcher, 2014, Vol.(78), № 7-1, p. 1237-1242.

9. V.Ya. Tsvetkov. Information interaction // European Researcher, 2013, Vol.(62), № 11-1, p. $2573-2577$.

10. Dyer J. Effective interfirm collaboration: how firms minimize transaction costs and maximize transaction value. 2002.

11. V.Y. Tsvetkov. Information Interaction as a Mechanism of Semantic Gap Elimination // European Researcher, 2013, Vol.(45), № 4-1, p. 782-786.

12. Tsvetkov V. Ya. Transaction costs in the implementation of information technology and systems // Modern Problems of Science and Education. 2008. №4. p. 114-116.

13. Obolyaeva N.M. Elimination of information asymmetries as a tool to improve the quality of education // News of higher educational institutions. Surveying and aerial photography. 2012. №6. p. 123-124.

14. Tsvetkov V.Ya. Information control. LAP LAMBERT Academic Publishing GmbH \& Co. KG, Saarbrücken, Germany 2012. 201 p.

15. Grossman S.J., Hart O.D. An analysis of the principal-agent problem // Econometrica: Journal of the Econometric Society. 1983. p. 7-45.

16. Cvitanić J., Zhang J. Principal-Agent Problem // Contract Theory in Continuous-Time Models. Springer Berlin Heidelberg, 2013. p. 3-6.

17. Holmstrom B., Milgrom P. Multitask principal-agent analyses: Incentive contracts, asset ownership, and job design // Journal of Law, Economics, \& Organization. 1991. p. 24-52.

18. V.Ya. Tsvetkov. Information Situation and Information Position as a Management Tool // European Researcher, 2012, Vol.(36), № 12-1, p. 2166-2170.

19. V.Ya. Tsvetkov. Multipurpose Management // European Journal of Economic Studies 2012, Vol.(2), № 2. p. 140-143.

20. Wen Y.M., Lu B.L. A cascade method for reducing training time and the number of support vectors //Advances in Neural Networks-ISNN 2004. Springer Berlin Heidelberg, 2004. p. $480-486$.

21. V.Ya. Tsvetkov. Complexity Index // European Journal of Technology and Design, 2013, Vol.(1), № 1. p.64-69.

22. Tsvetkov V.Ya. Analysis of tacit knowledge // Prospects of science and education 2014. №1. p.56-60. 
23. Tsvetkov V.Ya. Dichotomous Systemic Analysis. Life Science Journal 2014; 11(6). pp. 586-590.

УДК 332.01

\section{Информационная асимметрия как фактор рисков}

Виктор Яковлевич Цветков

Московский государственный технический университет радиотехники, электроники и автоматики (МГТУ МИРЭА), Российская Федерация

Доктор технических наук, профессор

E-mail: cvj2@mail.ru

Аннотация. Исследована информационная асимметрия как причина рисков при принятии решений. Описаны виды информационной асимметрии как фактора риска. Описаны виды риска, возникающие при разных информационных асимметриях. Описаны методы минимизации этих рисков. Раскрыта проблема агент принципал. Анализируются принципы минимизации приисков при возникновении этой проблемы. Показано применение специальных информационных моделей для минимизации рисков в этой проблеме. Описан каскадный метод минимизации риска принятия решений при информационной асимметрии.

Ключевые слова: управление; принятие решений; риски; информация; информационная асимметрия; принципал; информационное взаимодействие; минимизация рисков. 